\title{
The Edinburgh-Cape Blue Object Survey
}

\author{
R. S. Stobie, D. Kilkenny \\ South African Astronomical Observatory, PO Box 9, Observatory 7935, \\ Cape, South Africa
}

\section{O'Donoghue and A. Chen}

Department of Astronomy, University of Cape Town, Rondebosch 7700, South Africa

\begin{abstract}
The Edinburgh-Cape Blue Object Survey is a major survey to discover blue stellar objects brighter than B $\sim 18$ in the southern sky. It covers an area of sky of 10,000 square degrees with $|\mathrm{b}|>30^{\circ}$ and $\delta<0^{\circ}$. The blue stellar objects are selected by automatic techniques from U and B pairs of UK Schmidt Telescope plates scanned with the COSMOS measuring machine. Follow-up photometry and spectroscopy are being obtained with the SAAO telescopes to classify objects brighter than $B=16.5$. This paper describes the survey, the techniques used to extract the blue stellar objects, the photometric accuracy, and the completeness of the survey.
\end{abstract}

\section{Introduction}

The Palomar-Green Survey (Green, Schmidt \& Liebert 1986) carried out in the 1970s was a major impetus in leading to many new discoveries connected with hot ultraviolet-rich stars. This directly led to a north-south imbalance in the numbers of such objects. Many of the objects are intrinsically of great interest and in some cases sufficiently rare that only a handful of them are known. With these thoughts in mind it was expected that an extension of the Palomar-Green Survey to the southern hemisphere would yield equally rich returns.

The Edinburgh-Cape Blue Object Survey is a survey of $\sim 10,000$ square degrees at high galactic latitude in the southern hemisphere for ultraviolet-rich stellar objects. The motivation, technique and some initial results are described in Stobie et al. (1992) and Kilkenny et al. (1994). The primary categories of object which we expect to discover are hot $O$ and $B$ subdwarfs, white dwarfs, blue horizontal branch stars, B stars, cataclysmic variables and bright quasars.

\section{Selection of blue stellar objects}

$\mathrm{U}$ and B pairs of plates in each field are taken with the $1.2 \mathrm{~m}$ UK Schmidt Telescope in Australia. The U plate is a 60 minute exposure on $\mathrm{IaO}$ emulsion with a UG1 filter. The $\mathrm{B}$ plate is a 15 minute exposure on $\mathrm{IIaO}$ emulsion with 
a GG385 filter. The B plate is much deeper than the U plate, reaching B 20. This was deliberately chosen so that the star/galaxy discrimination would be reasonably reliable to $B \sim 18$. In order that the EC survey did not conflict with the primary surveys of the UK Schmidt Telescope it was decided that the plates would only be taken in non-survey conditions (i.e. seeing $>2$ arcseconds). In retrospect this was a fortunate decision because accurate wide-field stellar photometry is more difficult with plates taken in good seeing (assuming that one is not dealing with the crowded-field case). The reason is that in good seeing the image structure varies across a wide-angle photograph (Wallace \& Tritton 1979) resulting in systematically different isophotal magnitudes. Poor seeing blurs out these effects and makes uniform stellar photometry across a Schmidt plate easier to achieve.

All plates are digitised at the Royal Observatory, Edinburgh with the COSMOS machine (MacGillivray \& Stobie 1984) using the $16 \mu \mathrm{m}$ increment and the $32 \mu \mathrm{m}$ spot. The plates are physically of size $356 \times 356 \mathrm{~mm}^{2}$. For reasons of mechanical limitations the maximum area that COSMOS can measure is $287 \mathrm{x}$ $287 \mathrm{~mm}^{2}$. This area is not quite central on the plate but contains nearly all the unvignetted region of the UK Schmidt plate. With a plate scale of 67.12 arcsec $\mathrm{mm}^{-1}$ this corresponds to an area on the sky of $5.35 \times 5.35$ square degrees. With plate centres 5 degrees apart this ensures that there is complete sky coverage and a small degree of overlap. COSMOS outputs a catalogue of objects detected with a set of parameters describing each object. For this project only a subset of parameters was retained for subsequent processing, namely

$$
\begin{aligned}
& \text { B plate: RA, Dec, area, COSMAG, } \mathrm{I}_{s k y}, \mathrm{a}, \mathrm{b}, \theta, \mathrm{x}, \mathrm{y} \\
& \text { U plate: RA, Dec, area, COSMAG, } \mathrm{I}_{s k y}, \mathrm{a}, \mathrm{b}, \theta
\end{aligned}
$$

The RA and Dec are produced by automatically matching the brightest detected objects with the Smithsonian catalogue, given the RA, Dec of the plate centre. the plate scale and the plate orientation during measurement. These coordinates are accurate to 1 arcsec, and are required for both the pairing of the plates and the production of computer finding charts. The area is the number of pixels in the image. An area cut-off of $\mathbf{1 0}$ pixels was applied to avoid storage of images below this size. These images are in any case very faint (typically $B>20$ ) and of too low photometric accuracy for this project. The COSMAG parameter is defined as $-250 \log \Sigma_{i}\left(I_{i}-I_{s k y}\right)$ where the summation is over all pixels in the image and the intensities are relative intensities obtained from the step-wedge calibration. The semi-major axis, the semi-minor axis and orientation $(a, b, \theta)$ are obtained from fitting an ellipse to the second order unit-weighted moments (Stobie 1986) and were primarily used for the identification of elliptical images (usually galaxies or doubles). The $\mathrm{x}, \mathrm{y}$ centroids were retained to produce an $\mathrm{x}, \mathrm{y}$ plot to the scale of the Schmidt plate to aid in the visual inspection.

Using the RA and Dec coordinates with an error box of \pm 3 arcseconds the $\mathrm{U}$ and $\mathrm{B}$ plate catalogues were merged to produce a single catalogue retaining only objects which paired. Because the B plate was deeper the number of paired images was determined by the $U$ plate. The star/galaxy discrimination was determined from the B plate image parameters using a surface-brightness criterion (Fig. 1). The line drawn in Fig. 1 is defined relative to the stellar locus by a specified sigma cut. Objects below the line correspond to images of point sources and were classified stellar. Objects above the line are more extended than point 


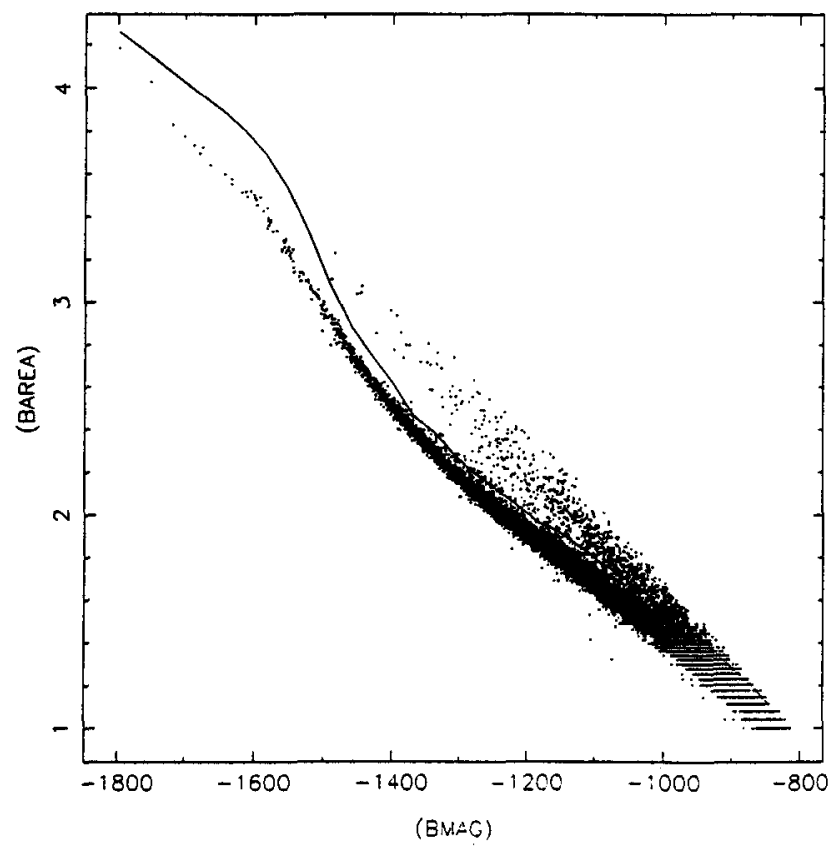

Figure 1. Star/galaxy discrimination using a surface brightness criterion. The ordinate is $\log ($ area $)$ where area is the number of pixels in the object. The abscissa is COSMAG which is related to the volume integral of the intensity above sky.

source images and were classified non-stellar. In practice the star/galaxy discrimination was effective to $B \sim 18$ (COSMAG -1000). Fainter than this it can be seen that the stellar and galaxian objects merge, and there is insufficient information on these plates to distinguish between them.

The selection of blue stellar objects was carried out in the natural system B, $\mathrm{U}-\mathrm{B}$ diagram (Fig. 2). At faint magnitudes the B plate limiting magnitude (10 pixel area cutoff) can be seen as an upper horizontal limit to the distribution of magnitudes. Similarly the U plate limiting magnitude can be seen as an upper sloping limit to the distribution of magnitudes, such that increasingly redder objects are lost at increasingly brighter B magnitudes. Fig. 2 shows an example of the selection polygon enclosing the interesting blue objects. The curvature in the $F / G$ type locus is a consequence of the non-linear response of the photographic emulsion and the limited density range of the COSMOS measuring machine. As these photographic magnitudes are not calibrated, the shape of this F/G star locus can vary from field to field. Nevertheless, at brighter magnitudes (COSMAG $<-1000$ ) there is a relatively sharp boundary between the F/G type stars and the bluer objects. The boundary, however, becomes less sharp at fainter magnitudes because the photometric errors are increasing. The number density of blue stellar objects selected varied as a function of galactic 


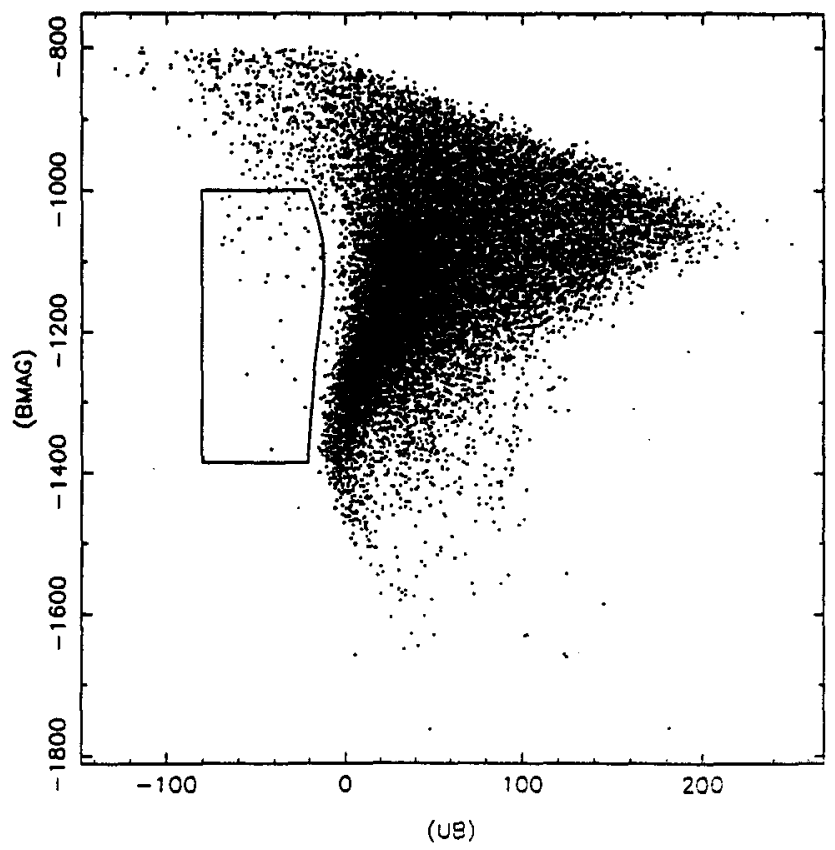

Figure 2. The selection of blue stellar objects in the natural system $\mathrm{B}, \mathrm{U}-\mathrm{B}$ colour-magnitude diagram. The polygon defines the objects of interest down to a faint magnitude of $B \sim 18$.

latitude and longitude with on average $\sim 50$ objects per field, corresponding to a number density of 2 per square degree.

\section{Photometry}

At this stage all we know is that the selected objects are blue and stellar. In order to classify the objects, follow-up photometry and spectroscopy brighter than B $=16.5$ is being obtained at the South African Astronomical Observatory.

\subsection{Photoelectric UBV photometry}

The (U-B, B-V) two colour diagram is shown in Fig. 3 for a sample of stars in the survey. The two-colour diagram clearly identifies a number of subgroups. The $F / G$ type subdwarfs fall within a boundary stretching from the Hyades main sequence to an upper locus defined by the most metal poor subdwarfs. One of the uses of the photometry is that any star falling in this domain is classed as an F/G subdwarf and usually no further photometry or spectroscopy of it is obtained. Another group of stars are the blue horizontal branch stars and normal B stars. By far the most common type of object (accounting for $\sim 50 \%$ of the survey) are the hot $\mathrm{O}$ and $\mathrm{B}$ subdwarfs. These stars cover a wide range 


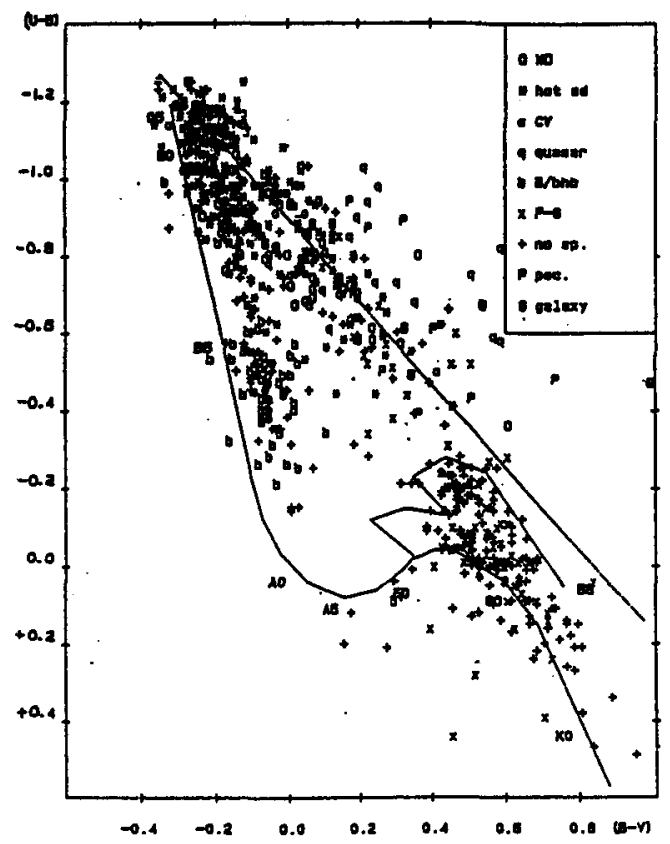

Figure 3. The (U-B, B-V) two-colour diagram for candidate blue objects with spectroscopic classification.

of temperatures which are so hot that the UBV photometric system provides no discrimination in temperature. Spectroscopy is essential to distinguish the different types of $\mathrm{O} / \mathrm{B}$ subdwarf with differing temperature, gravity and chemical abundance. The remaining objects tend to cluster around or above the black body line and are a diverse group comprising white dwarfs, cataclysmic variables, quasars and emission-line galaxies.

\subsection{Accuracy of the $U$ and $B$ photographic photometry}

The completeness of the catalogue of ultraviolet-rich stars depends critically on the accuracy of the photographic U-B colour measurement. Investigation of this is possible using the UBV photoelectric photometry with which the photographic photometry can be calibrated. Previous experience with wide-angle Schmidt photographs and automated measuring machines would lead one to expect errors in U, B photographic magnitudes of $\sim 0.1 \mathrm{mag}$ for isolated stellar images more than 2 mag above the plate limit (Reid \& Gilmore 1982, Stobie \& Ishida 1987). This leads to an expected accuracy of $\sim 0.15$ mag for the photographic (U-B) colour.

O'Donoghue et al. (1993) have assessed the accuracy of the photographic photometry in the EC Survey on the basis of almost 90 fields for which photoelectric photometry is complete to $\mathrm{B} \sim 16.5$. For each of these fields, plots of photographic photometry (hereafter called Cos U and Cos B) versus photo- 


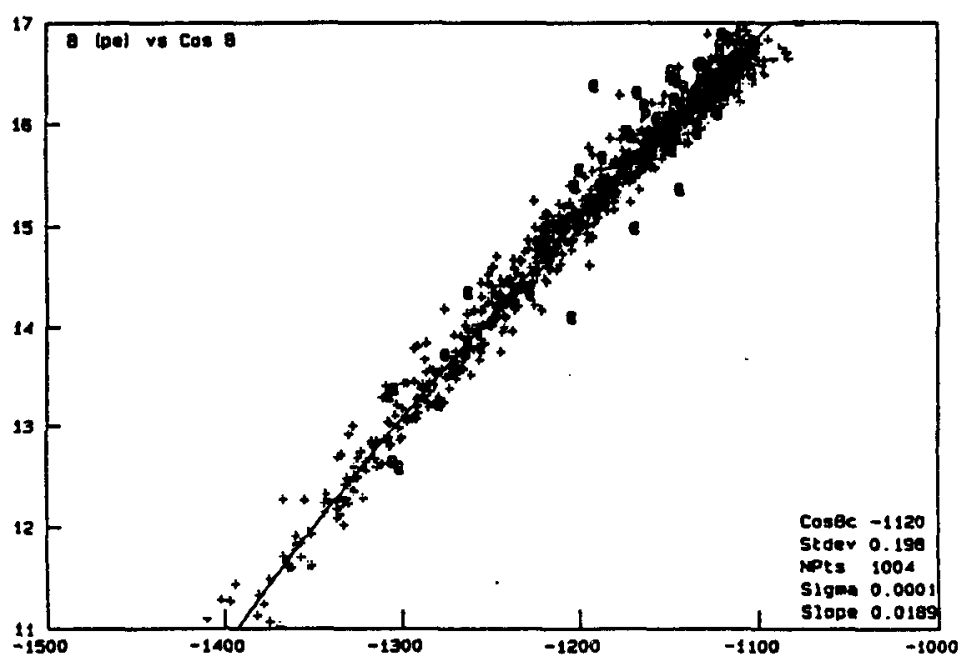

Figure 4. Combined plot of $\mathrm{B}_{p e}$ (ordinate) vs. Cos $\mathrm{B}$ (abscissa).

electric photometry (called $\mathrm{U}_{p e}$ and $\mathrm{B}_{p e}$ respectively) were produced. For some fields, tight parabolic relationships between $\operatorname{Cos} \mathrm{U}$ and $\mathrm{U}_{p e}$, and $\operatorname{Cos} \mathrm{B}$ and $\mathrm{B}_{p e}$ were found, with residual standard deviations of $\sim 0.1 \mathrm{mag}$. For the majority of fields, however, the residual scatter was much worse: $0.2 \mathrm{mag}$ typically but occasionally reaching as much as $0.3-0.4 \mathrm{mag}$. This result would seem to imply that Cos $\mathrm{U}-\mathrm{B}$ is measured with an accuracy of $0.3 \mathrm{mag}$ or worse!

For most fields the number of stars that could be used in the calibration was less than 15 and known variables amongst the blue objects caused problems. In order to gain an overall assessment of the accuracy of the photographic photometry, the results for all the completed fields were combined. This necessitated determining the arbitrary zero point for each field by fitting a parabola with fixed linear and quadratic coefficients to the plots of Cos B vs. $B_{p e}$ (and Cos $\mathrm{U}$ vs. $\left.\mathrm{U}_{p e}\right)$. This zero point was then subtracted from all the stars within the corresponding field allowing a single plot of Cos B vs. $B_{p e}$ (Fig. 4) or Cos U vs. $U_{p e}$ to be made. The scatter in the residuals about the best-fitting parabola was found to be $0.20 \mathrm{mag}$ for $\operatorname{Cos} \mathrm{B}$ and $0.30 \mathrm{mag}$ for Cos $\mathrm{U}$, with most of the additional scatter in Cos $\mathrm{U}$ coming from the sdF and sdG stars with $\mathrm{U}-\mathrm{B}>$ -0.2 . This additional scatter is not random: the residuals of the $s d F$ and $s d G$ stars are systematically biased towards brighter values of Cos $U$ than implied by their values of $\mathrm{U}_{\text {pe }}$.

The presence of these sdF and sdG stars is clearly seen in the two-colour diagram for a sample of stars in the survey with photoelectric photometry (Fig. 3). It is apparent in this diagram that there is a dearth of horizontal branch $\mathrm{A}$ stars $\left(\mathrm{B}-\mathrm{V}_{p e}=\mathrm{U}-\mathrm{B}_{p e}=\sim 0.0\right)$, but significant contamination $(\sim 20 \%)$ of the survey sample by $\mathrm{sdF}$ and sdG stars $\left(\mathrm{U}-\mathrm{B}_{p e} \geq-0.2, \mathrm{~B}-\mathrm{V}_{p e} \geq 0.4\right)$. Taken at face value, this seems to imply that there is a strong colour term in the relation 
between $\operatorname{Cos} \mathrm{U}-\mathrm{B}$ and $\mathrm{U}-\mathrm{B}_{p e}$, which results in objects which are redder in $\mathrm{B}-\mathrm{V}$ being selected at redder $\mathrm{U}-\mathrm{B}_{p e}$.

Considerable effort was expended in trying to find a colour term in the relationship between $\mathrm{Cos} U$ and $\mathrm{U}_{p e}$ which would account for the contamination of the survey sample by red subdwarfs. However, plots of the residuals vs. colour in the Cos $U$ and $\mathrm{U}_{p e}$ relation yielded no consistent colour term. This failure to find a colour term agrees with the colour equations derived by Blair and Gilmore (1982) for the combination of UK Schmidt Telescope, filters and IlaO emulsion used in the EC Survey.

\subsection{Non-Gaussian errors in photographic photometry}

It has long been recognised that the error distribution in photographic photometry is non-Gaussian (Gilmore 1983) and shows an extended tail. This is an important effect if one is carrying out a photographic survey for objects of extreme colour or unusual properties, such as variables, as the non-Gaussian tail leads to a small fraction of "normal" stars contaminating the desired sample with spurious candidates.

By assuming that the error distribution of photographic magnitudes in the EC survey fields is the same as that determined by Stobie and Henley (1983), and that the U-B distribution of stars is the same as that determined by Yoshii, Ishida and Stobie (1987), estimates have been made of the number of sdF and sdG stars scattered into the survey sample by an apparent, but spurious, blue U-B colour. Because the $F / G$ stars so outnumber the intrinsically blue stars only a small percentage of non-Gaussian errors can cause significant contamination. Detailed calculations have shown that the observed $\sim 20 \%$ contamination of $\mathrm{F} / \mathrm{G}$ stars in the survey can be explained entirely by the non-Gaussian error distribution.

This result also explains why the $\mathrm{sdF}$ and sdG stars affect the Cos $\mathrm{U}$ photometry only: those objects showing anomalously bright Cos B values would not be selected by the survey technique.

\subsection{Accuracy of U-B photographic colour and correlated errors}

With the recognition that the $\mathrm{sdF}$ and $\mathrm{sdG}$ stars contaminating the sample have anomalously large errors in the photographic photometry, it was clear that the calculations of section 3.2 had to be repeated after excluding all objects with $\mathrm{U}-\mathrm{B}_{p e} \geq-0.3$. The result for the $\mathrm{B}$ mag calibration is similar to that obtained before, with the standard deviation of the residuals, $0.20 \mathrm{mag}$, only showing a slight improvement by the exclusion of the red subdwarfs. The $\mathrm{U}$ mag calibration showed a similar plot to the B mag calibration but now with a much smaller scatter, $0.22 \mathrm{mag}$, in comparison to the previous value of $0.30 \mathrm{mag}$ when the red subdwarfs were not excluded.

The most interesting relationship is between Cos $\mathrm{U}-\mathrm{B}$ and $\mathrm{U}-\mathrm{B}_{p e}$ which is shown in Fig. 5. The standard deviation of the residuals about the best linear fit is only $0.16 \mathrm{mag}$, smaller than the errors in Cos $U$ or Cos B, and much smaller than the value of $0.30 \mathrm{mag}$ obtained by adding the errors in quadrature. The reason for the smaller error in Cos $\mathrm{U}-\mathrm{B}$ is simply that the residuals in Cos $\mathrm{U}$ and Cos B are correlated. This is clearly illustrated in Fig. 6 which shows a plot 


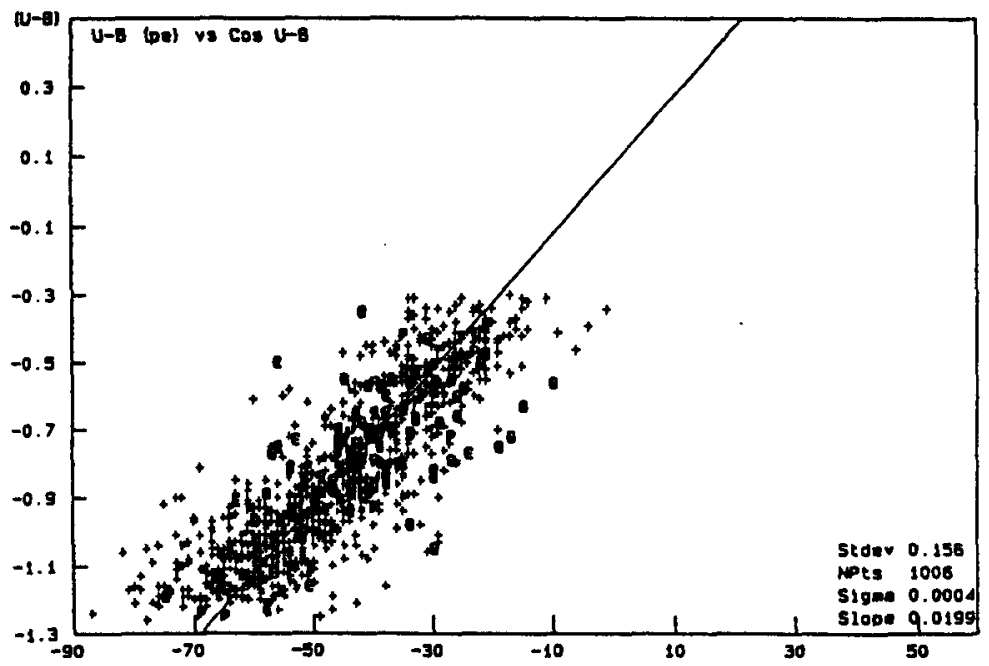

Figure 5. Combined plot of $U-B_{p e}$ (ordinate) vs. Cos U-B (abscissa).

of the residuals of the Cos B vs. $B_{p e}$ relation (Fig. 4) against those of the Cos $\mathrm{U}$ vs. $\mathrm{U}_{p e}$ relation.

The strong correlation of the errors in Fig. 6 is obviously intimately associated with the cause of the disturbingly large scatter in the Cos B vs. $B_{p e}$ and Cos $U$ vs. $U_{p e}$ relations. In this connection, it is important to remember that the $U$ and $B$ photography is done on different plates but as close together in time as possible. Strenuous attempts have been made to identify the cause of these correlations, so far without success. The errors in the individual magnitudes do not correlate with apparent magnitude, colour or position on the plate. Nor do the errors correlate with the quality of the seeing as might be expected if variations of image profile across the wide-angle photograph were a major source of error.

At present the explanation for the large and correlated errors is unknown. However, without such a correlation the survey would not work so well. Blue stellar objects in the EC survey are being selected with an accuracy in photographic $U-B$ of $0.16 \mathrm{mag}(1 \sigma)$. This result compares favourably with the corresponding value of $0.38 \mathrm{mag}$ for the Palomar-Green survey (Green, Schmidt \& Liebert 1986). The individual photographic magnitudes, however, are being measured with lower accuracy $(\sim 0.20 \mathrm{mag})$ than the $(\mathrm{U}-\mathrm{B})$ colour.

\section{Blue object classification}

A spectrogram is being obtained for every object brighter than $B=16.5$ provided the UBV photometry, if available, did not place the object in the $F / G$ subdwarf region of the two-colour diagram. Each spectrogram is obtained with the grating spectrograph and intensified Reticon photon counting system on the SAAO $1.9 \mathrm{~m}$ 


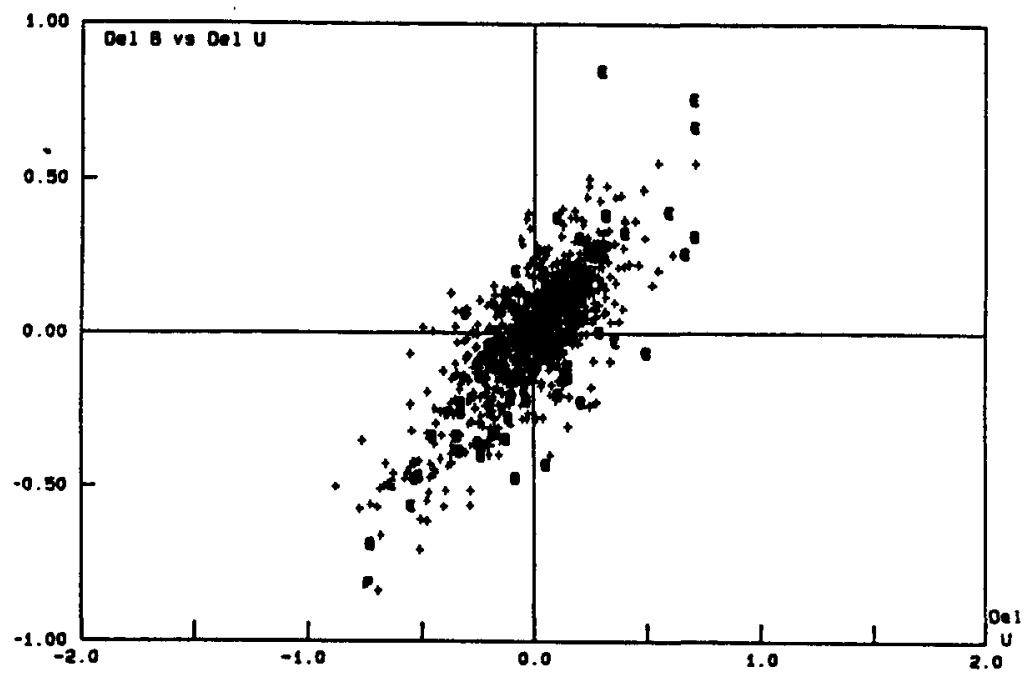

Figure 6. Correlation of residuals in $\operatorname{Cos} B$ (ordinate) vs. residuals in Cos U (abscissa).

telescope. Grating 6, giving a reciprocal dispersion of $100 \AA \mathrm{mm}^{-1}$ and blazed to be optimum at $4300 \AA$ was used for all spectroscopy. With a normal slit width of $250 \mu \mathrm{m}$ corresponding to 1.8 arcseconds, the effective resolution was $\sim 3.5 \AA$. The spectral range covered was $3400 \AA<\lambda<5400 \AA$, though because of the fall-off in the intensification chain at the edges the useful wavelength range was limited to $3600 \AA<\lambda<5200 \AA$.

Except for the identification of $F / G$ subdwarfs, the spectroscopy is essential for the classification of the type of blue object. A description of the spectral classification procedure is given in Kilkenny et al. (1994) and examples of spectra in Stobie et al. (1992).

\section{Completeness of survey}

The internal completeness of the survey can be assessed in two ways (i) by comparing the results for duplicate U,B plate pairs of the same field and (ii) by examining the overlap region between plate pairs in different fields.

Duplicate plate pairs exist for three fields. The total number of blue objects identified and the number in common does not give a true measure of the completeness statistics as the magnitude limits may differ between the two selections and the colour position of the red boundary will not be identical. Thus the statistics have been restricted to objects with $B<16.5$ as these are objects for which we have both photometry and spectroscopy. This also enables us to examine the spectral type of the objects not in common. The comparison reveals that the objects not in common are nearly all $F / G$-type stars with a few early-type stars. This shows that objects of spectral type earlier than F/G are 


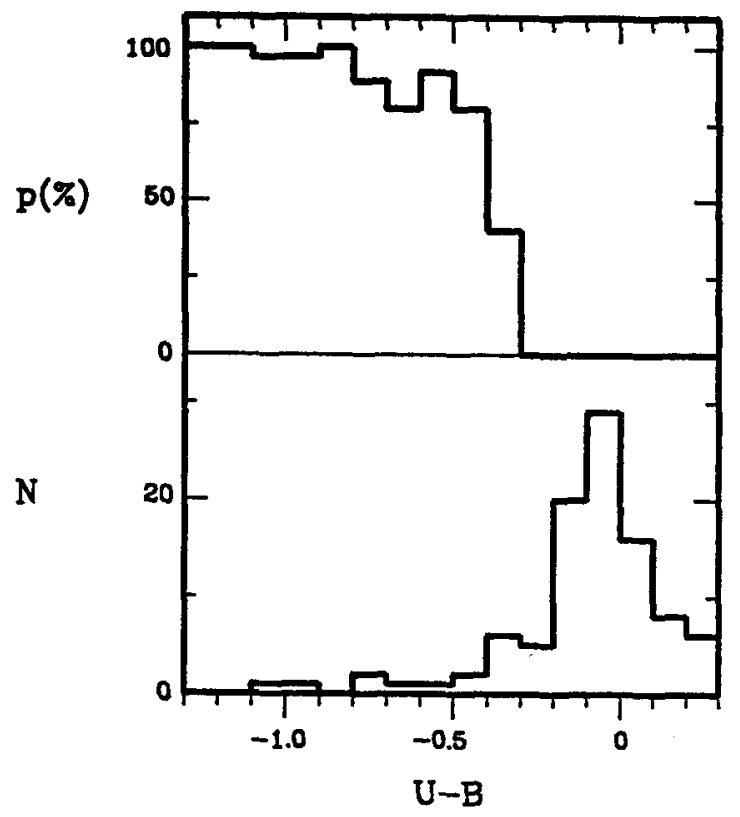

Figure 7. From the plate overlap regions (a) the percentage of objects in common (b) the number of objects found only once, both as a function of the photoelectric $U-B$ colour.

found to be common to both selections and the objects not in common are by and large stars of later spectral type. Thus the main reason for the difference in blue object selection is the non-Gaussian error distribution in photographic magnitudes (section 3.3) causing a small percentage of $F / G$ type stars to be selected spuriously as candidate blue stellar objects.

The second method of determining the completeness was to examine the regions of plate overlap in different fields. The boundaries of plate measurement are defined in $\mathrm{x}, \mathrm{y}$-space by COSMOS (and this region is not centred on the plate). The $x, y$ to RA,Dec transformation enables the boundaries of the measured area to be defined in celestial coordinates. From this one can then calculate the overlap region between neighbouring fields. The blue object selection in this overlap region was then compared for the common objects and non-common objects. Provided we only considered fields with UBV photoelectric photometry and objects brighter than $B=16.5$ this enabled the completeness of the survey to be defined as a function of $\mathrm{U}-\mathrm{B}$.

Fig. 7 illustrates this percentage completeness based on the objects found twice in the overlap region and the number of objects found only once in the overlap region, both as a function of $\mathrm{U}-\mathrm{B}$ colour. It is clear that the objects found only once are predominantly redder, as one would expect, since the selection of these objects is sensitive not only to the non-Gaussian errors in magnitudes but also to the location of the red boundary, which may vary slightly from field to field (as it is determined from the uncalibrated photographic magnitudes). It is also apparent that the effective colour cutoff for the survey is $\mathrm{U}-\mathrm{B}^{\circ}<-0.4$. 
Bluer than this the survey is $93 \%$ complete. It is noticeable that a small percentage of very blue objects (e.g. $U-B<-1.0$ ) is found only once. The reasons for this are being followed up.

\section{Acknowledgments}

The achievement of a survey of such large scale would not be possible without the support of many dedicated staff. We especially wish to express our appreciation to the staff of the UK Schmidt Telescope in Australia for taking such high quality photographic plates, to the staff of the COSMOS facility in Edinburgh for the excellence of the automated measuring machine and to Prof M. W. Feast, the former Director of SAAO, for allocating substantial amounts of telescope time for the follow-up photometry and spectroscopy.

\section{References}

Blair M. \& Gilmore G., 1982, PASP, 94, 742

Gilmore G., 1983, in Workshop on Astronomical Measuring Machines, R. S. Stobie \& B. McInnes, eds, Occ. Rep. R. Obs. Edinburgh, 10, 259

Green R. F., Schmidt M. \& Liebert J., 1986, ApJS, 61, 305

Kilkenny D., O'Donoghue D., Stobie R. S., Chen A., Koen C \& Savage A., 1994, in Hot Stars in the Halo, (Schenectady NY), in press

MacGillivray H. T. \& Stobie R. S., 1984, Vistas in Astronomy, 27, 433

O'Donoghue D., Stobie R. S., Chen A., Kilkenny D. \& Koen C., 1993, in Precision Photometry, D. Kilkenny, E. Lastovica \& J. M. Menzies, eds, (South African Astronomical Observatory), p. 72

Reid N. \& Gilmore G., 1982, MNRAS, 201, 73

Stobie R. S., 1986, Pattern Recognition Letters, 4, 317

Stobie R. S., Chen A., O'Donoghue D. \& Kilkenny D., 1992, in Variable Stars and Galaxies, ASP Conf. Ser. 30, B. Warner, ed., 87

Stobie R. S. \& Henley R. C., 1983, in Workshop on Astronomical Measuring Machines, R. S. Stobie \& B. McInnes, eds, Occ. Rep. R. Obs. Edinburgh, 10,49

Stobie R. S. \& Ishida K., 1987, AJ, 93, 624

Wallace P. T. \& Tritton K. P., 1979, MNRAS, 189, 115

Yoshii Y., Ishida K. \& Stobie R. S., 1987, AJ, 93, 323

\section{Discussion}

Hawkins: The correlation of $\mathrm{U}-\mathrm{B}$ errors sounds as if it might be caused by position dependent errors. Also, how do you establish your magnitude limit of $\mathrm{B}=16.5$ without calibration?

Stobie: We have examined the correlated $\mathrm{U}-\mathrm{B}$ residuals with $\mathrm{x}, \mathrm{y}$-positions on plates and $r, \theta$-positions. No dependences were found. The magnitude limit of 
$\mathrm{B}=16.5$ is obtained by ordering stars in decreasing brightness from COSMOS magnitudes and observing photoelectrically until we reach $B=16.5$.

Parker: How seriously would the aims of the survey be compromised if lcak of IIaO material caused early curtailment of the survey?

Stobie: For projects that depend on completeness of the survey (e.g. distribution of sub-dwarf $\mathrm{O} / \mathrm{B}$ stars as a function of galactic latitude and longitude) this will be a serious effect. For the discovery of interesting stellar objects, it will simply mean that we do not find as many. We would prefer to complete the survey, with a different emulsion, if necessary. 